\title{
Utility of Chromogenic Medium in Characterization of Enterococci in Urinary Tract Infection and Phenotypic Detection of Their Virulence Factors
}

\author{
Betu Rama Soujanya ${ }^{1}$, Banashankari G S ${ }^{2}$ \\ ${ }^{1}$ Postgraduate, Department of Microbiology, M S Ramaiah Medical College, Bangalore, Karnataka, India. \\ ${ }^{2}$ Professor, Department of Microbiology, M S Ramaiah Medical College, Bangalore, Karnataka, India.
}

Corresponding Author: Betu Rama Soujanya

\begin{abstract}
Introduction: Enterococci from being intestinal commensals have evolved in becoming pathogens and are associated with significant morbidity and mortality

Aims \& Objectives: This study was done to speciate the uropathogenic Enterococci using the chromogenic medium and to determine the antibiogram also to detect virulence factors phenotypically.

Materials and methods: The study included a total of 30 uropathogenic Enterococci isolated over 6 months. Speciation was done using HiCrome Enterococcus faecium agar base. Antibiotic sensitivity was done by the Kirby Bauer Disc Diffusion method as per Clinical and Laboratory Standards Institute (CLSI) guidelines. Among the virulence factors hemolysin, haemagglutination, and gelatin liquefaction tests were done.

Results: Amongst the 30 enterococci isolates, 17 were Enterococcus faecalis (E. faecalis) $(56.66 \%)$ \& 13 were Enterococcus faecium (E. faecium) (43.33 \%). $100 \%$ of the Enterococcus species were sensitive to Vancomycin \& Teicoplanin. $66.67 \%$ of the Enterococci showed hemolysis, $10 \%$ haemagglutination, and $43.33 \%$ gelatinase property.

Conclusion: Most common isolated species were Enterococcus faecalis. The changing patterns of antibiotic sensitivity to Enterococci in patients with urinary tract infection possess difficulty in selection of the antibiotics. Failure to synergistic therapy is seen in cases of resistance to High-level Gentamicin. Therefore, speciation and antibiotic sensitivity patterns will help in setting up an empirical therapy and thereby help in the reduction of morbidity and mortality.
\end{abstract}

Keywords: Antibiotic susceptibility, Chrome agar, Enterococcus species, virulence factors.

\section{INTRODUCTION}

- Enterococci are a part of the normal enteric microbiota that has evolved in becoming pathogens. They are among the most frequent causes of healthcareassociated infections transmitted from one patient to another primarily on the hands of hospital personnel, some of whom may carry them in their gastrointestinal tracts. Enterococci occasionally are transmitted on medical devices. The most common sites of infection are the urinary tract, wounds, biliary tract, and blood ${ }^{[1]}$.

- Patients who have been hospitalized for extended periods receiving broadspectrum antibiotics and elderly patients are more prone to Enterococcal infection [2].

\section{Aims \& objectives:}

- To speciate the Enterococci using the chromogenic medium.

- To determine the antibiogram of the uropathogenic Enterococci. 
- Phenotypic detection of virulence factors

\section{MATERIALS AND METHODS}

- This is a prospective study conducted in a tertiary care hospital over 6 months which included a total of 30 uropathogenic Enterococci isolates.

- Urine samples were inoculated on Cysteine lactose electrolyte deficient (CLED) agar by semi-quantitative method with a sterile standard loop and incubated at $37^{\circ} \mathrm{C}{ }^{[3]}$. Isolates were spot inoculated on HiCrome Enterococcus faecium agar base and incubated at $37^{\circ}$ C. Reading was taken after 24 hours to look for a change in color of the isolate. A sugar fermentation test was also done for the above isolates. Antibiotic sensitivity was done by Kirby Bauer Disc Diffusion method on Mueller Hinton agar as per Clinical Laboratory Standards Institute (CLSI) guidelines ${ }^{[4]}$.

- The antibiotics tested were Ampicillin $(10 \mu \mathrm{g})$, High- level Gentamicin $(120 \mu \mathrm{g})$, Ciprofloxacin $(5 \mu \mathrm{g})$, Linezolid $(30 \mu \mathrm{g}), \quad$ Teicoplanin $(30 \mu \mathrm{g})$ and Nitrofurantoin $(300 \mu \mathrm{g})$.

- Haemolysin production: The isolates were inoculated on 5\% sheep blood agar and incubated overnight at $37^{\circ} \mathrm{C}{ }^{[5]}$.

- Haemagglutination: This was performed as a direct bacterial haemagglutination test - slide method [6].

$>$ The isolates were inoculated in nutrient broth at $37^{\circ} \mathrm{C}$ for 48 hours for full fimbriation. The human blood group "O" $\mathrm{RBC}$ was taken and washed thrice with normal saline. After that, a final $3 \%$ suspension of $\mathrm{RBC}$ is prepared with fresh normal saline. A drop of broth culture and a drop of $3 \%$ RBC suspension is added on the slide and rocked at room temperature for 5 minutes.

- Positive: Presence of clumping seen.

- Negative: Absence of clumping
- Gelatinase Test: The production of gelatinase by the organisms was tested by the stab method ${ }^{[7]}$.

$>$ Tubes containing Nutrient gelatin medium were stabbed 4- 5 times with heavy inoculum and incubated for 48 hours at $37^{\circ} \mathrm{C}$. After 48 hours, tubes were placed in the refrigerator at $4^{\circ} \mathrm{C}$ for 30 minutes and observed for liquefaction of medium.

\section{RESULTS}

Total Enterococci isolated in pure cultures were 30 .

All the isolates were spot inoculated on HiCrome agar medium and incubated at $37^{\circ} \mathrm{C}$. All the E.faecium species fermented arabinose and produced green-colored colonies along with yellow coloration to the medium (Figure 1).

Speciation based on sugar fermentation and HiCrome agar medium showed the same result.

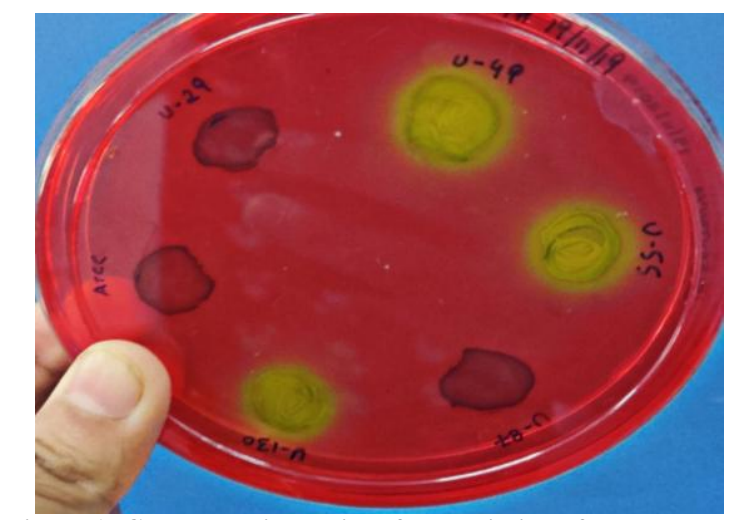

Figure 1: Chromogenic medium for speciation of Enterococcus E. faecalis: blue color. E. faecium: green colored colony with a yellow coloration of the medium

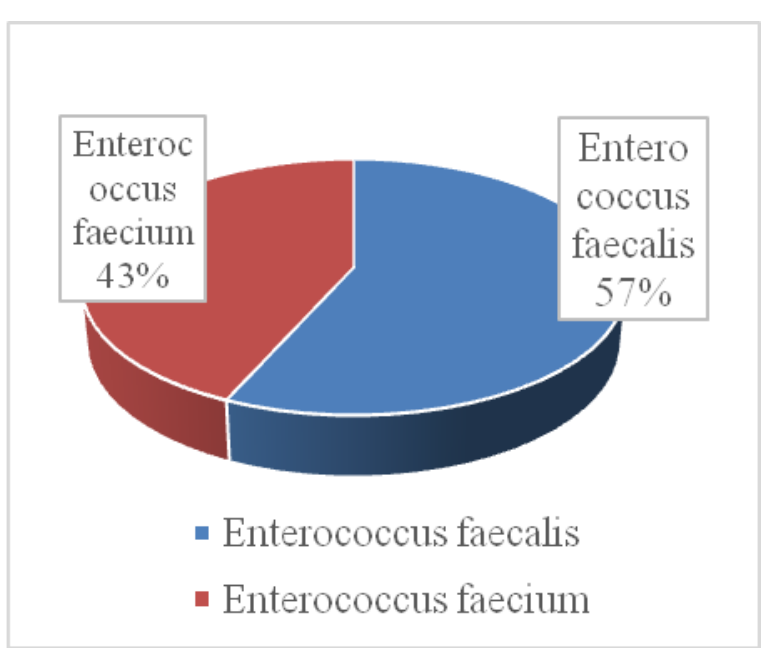

Figure 2: Distribution of species of Enterococcus 
Betu Rama Soujanya et.al. Utility of chromogenic medium in characterization of enterococci in urinary tract infection and phenotypic detection of their virulence factors.

Based on the chromogenic medium, it was observed that $57 \%$ were E.faecalis followed by $43 \%$ of E.faecium (Figure 2)

All the isolated Enterococcus species were phenotypically tested for the virulence factors like hemolysin production, hemagglutination, and gelatinase property. It was observed that $66.67 \%$ showed hemolysis property followed by $43.33 \%$ gelatinase property and only $10 \%$ showed hemagglutination property (Figure 3 ).

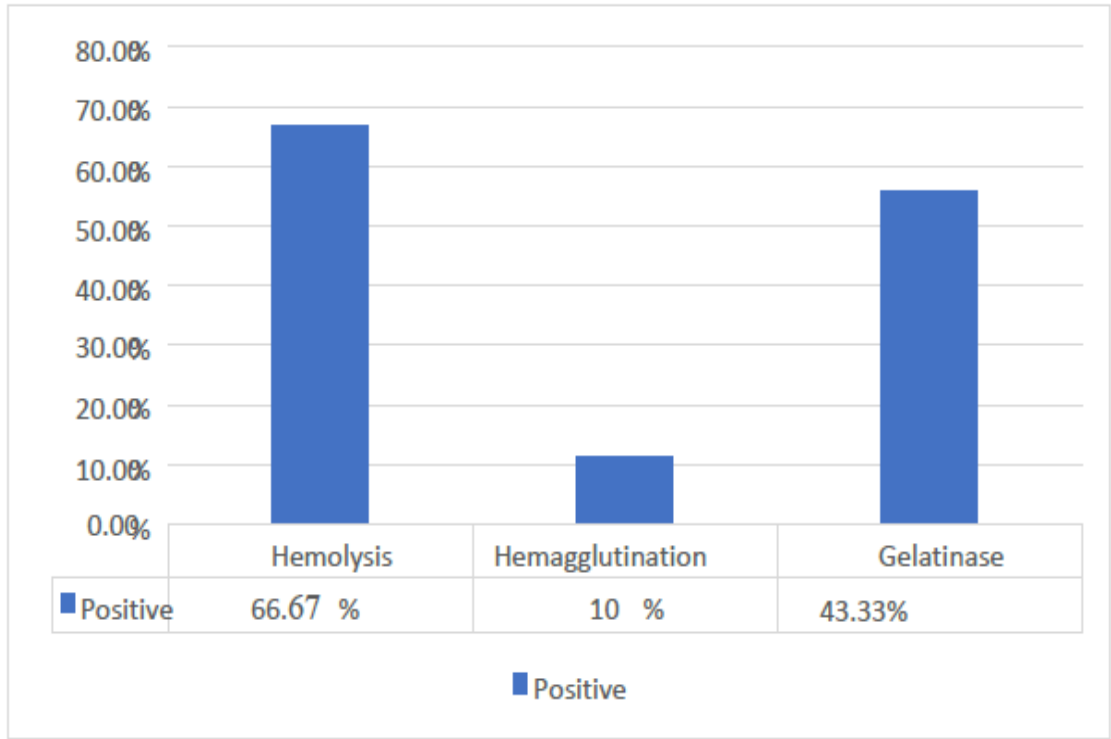

Figure 3: Distribution of Virulence factors of Enterococcus species $(\mathbf{n}=\mathbf{3 0})$

Amongst the two species, E.faecium showed $84.62 \%$ of hemolysis and $38.46 \%$ of gelatinase property (Figure 4).

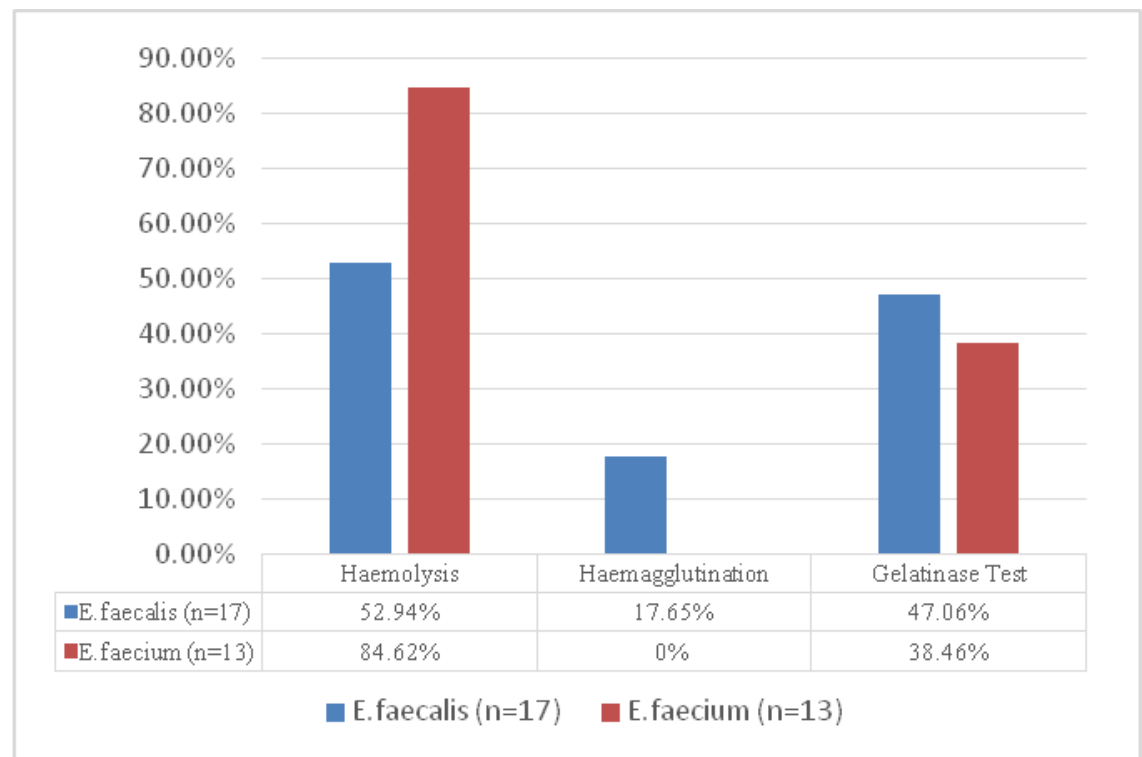

Figure 4: Distribution of Virulence factors amongst Enterococcus species

Both the species of Enterococcus were $100 \%$ susceptible to Vancomycin and Linezolid. However, $76.47 \%$ E.faecalis and
$61.54 \%$ of E. faecium were susceptible to Nitrofurantoin (Figure 5). 


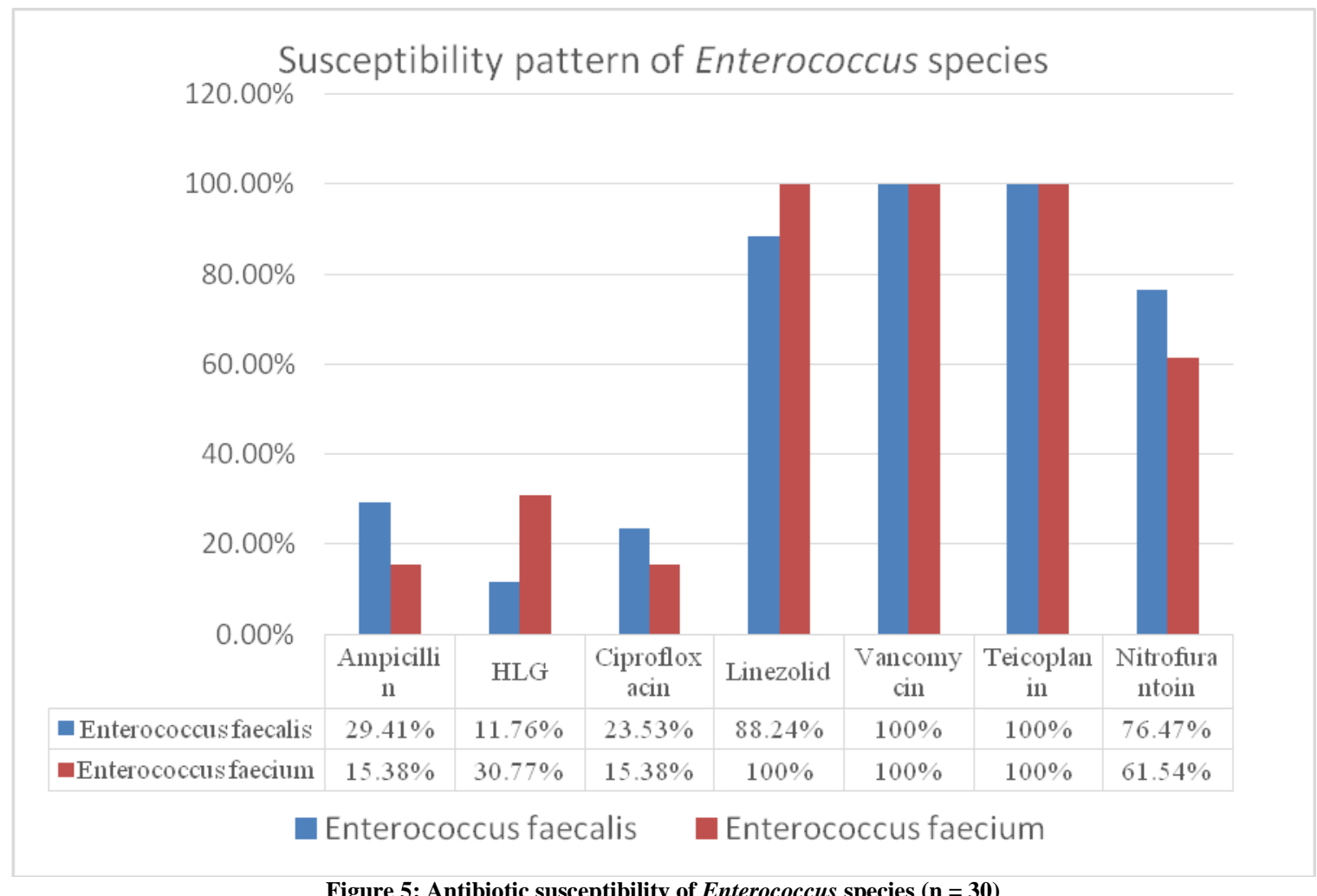

\section{DISCUSSION}

One of the most common causes of nosocomial and community-acquired infections are urinary tract infections (UTIs) affecting people of all ages and sex worldwide. Bacteria are the most common etiological agents causing UTI followed by fungal or very rarely a viral agent. Among the bacteria, $90 \%$ of the UTIs are caused by Gram-negative organisms followed by $10 \%$ of Gram-positive bacteria ${ }^{[8]}$.

Enterococcus species are Grampositive coccus which is commensals in the oral cavity, gastrointestinal tract, and vaginal vault, but can also act as pathogens by adhering and invading the host tissue and cause a wide variety of infections in humans. Enterococcus species are the third leading cause of hospital-acquired UTIs and constitute $15-30 \%$ of catheter-associated UTIs and are also responsible for community-acquired UTI ${ }^{[9]}$. Enterococcus faecalis causes 80 to $90 \%$ of human enterococcal infections, while the rest is caused by E $_{\text {faecium }}{ }^{[10]}$.

In this present study, HiCrome Enterococcus faecium agar was used for speciation of the species which utilizes the possession of the enzyme $\beta$-glucosidase, which specifically cleaves the chromogenic substrate to produce blue-colored colonies. E. faecium ferment arabinose; and cleaves the chromogenic substrate present in the media to produce green-colored colonies along with yellow coloration to the medium. E. faecalis does not ferment arabinose and therefore retains the blue color. Out of 30 isolates of Enterococcus 17 were E. faecalis and 13 were E. faecium. This chromogenic medium might help us in better speciation compared to the use of other biochemical reactions (sugar fermentation test)

Some of the virulence factors expressed by these enterococci species to evade the immune response and cause the infection are cytolysin or hemolysin, protease or gelatinase adhesins, aggregation substance, enterococcus surface protein(esp), etc. ${ }^{[10,11,12]}$.

In our study, it was noted that $80 \%$ of the isolates were from inpatients and $20 \%$ from outpatients.

In our study, hemolysin, gelatinase, and haemagglutination properties were tested phenotypically. 
Hemolysin is cytolytic which acts as a colonization factor and strains with cytolytic activity express a bacteriocin that is active against other Gram-positive organisms. In the present study, it was observed that $66.67 \%$ isolated produced hemolysin. In other studies, like Das AK et al., Banerjee $\mathrm{T}$ et al., and Ira $\mathrm{P}$ et al., reported being 38\%, 31.61\%, $21 \%$ respectively ${ }^{[13,14,15]}$.

The hemagglutination property helps the strains to adhere to the host urothelial cells. There are different adhesion molecules and they vary in the agglutination property to RBC of different species. In the present study, haemagglutination property was seen in only $10 \%$ of the isolates. Banerjee $\mathrm{T}$ et al., ${ }^{[14]}$ and Das AK et al., ${ }^{[13]}$ noted it to be $21.93 \%$ and $14 \%$ respectively.

Gelatinase is metalloendopeptidase II which has the property to break down and invade the host tissue by hydrolyzing gelatin, collagen, etc. In the present study, it was noted that $43.33 \%$ of isolates showed gelatinase production. Other studies like Das AK et al ${ }^{[13]}$., Ira P et al ${ }^{[15]}$., Banerjee $\mathrm{T}$ et al ${ }^{[14]}$., reported $66 \%, 19 \%$, and $9.03 \%$ respectively.

Antibiotic susceptibility of Enterococcus species showed that $100 \%$ of the isolates were susceptible to Vancomycin \& Teicoplanin, followed by Linezolid $(93.33 \%)$ and Nitrofurantoin $(70 \%)$. In the present study, only $23.33 \%$ of isolates were susceptible to ampicillin followed by $20 \%$ isolates were susceptible to Ciprofloxacin and high-level gentamicin.

Thattil et al ${ }^{[16]}$ had reported susceptibility to Vancomycin, Teicoplanin, Linezolid, Ciprofloxacin to be $82.2 \%$, $88.5 \%, 86.7 \% 51.3 \%$ respectively.

Shivani et al ${ }^{[17]}$ reported $100 \%$ susceptibility to Vancomycin and Linezolid with $69.2 \%$ isolates susceptible to Nitrofurantoin.

Amit A. Rangari et al ${ }^{[18]}$ reported 93.33\% isolates susceptible to Linezolid, $91.67 \%$ isolates to Nitrofurantoin, and only $6.67 \%$ isolates to Ciprofloxacin.
In the present study, it was noted that E. faecium was more drug-resistant compared to E. faecalis.

\section{CONCLUSION}

Enterococcus faecalis was the most common species isolated among Enterococci. Chrome agar can also be used in par with biochemical reactions to speciate Enterococci which has a better visual effect than the biochemical reactions (sugar fermentation). There is a need for speciation and antibiotic susceptibility testing due to the emergence of E. faecium strains and the development of drug resistance which might help the clinicians to start the patient on empirical therapy and thereby help in the reduction of morbidity and mortality.

Conflict of Interest: The authors declare that they have no competing interests.

\section{Financial Support and Sponsorship: Nil}

\section{Has The Ethical Clearance Obtained From Your Institution? YES}

CONSENT: A written informed consent was taken from all the patients.

\section{Acknowledgement: None}

\section{REFERENCES}

1. Karen C.Caroll, Janet S. Butel, Stephen A. Morse, Timothy Mietzner: Jawetz, Melnick \& Adelberg's Medical Microbiology : $27^{\text {th }}$ Edition

2. Murray, Rosenthal, Pfaller: Medical Microbiology: $8^{\text {th }}$ Edition.

3. Siva Prasad Reddy Basava. 2016. Isolation and Antibiogram of Enterococci from Patients with Urinary Tract Infection in a Tertiary Care Hospital. Int.J.Curr. Microbiol. App.Sci. 5(8): 658662.

4. CLSI M100: Performance Standards for Antimicrobial Susceptibility Testing.29th edition

5. Mittal S, Sharma M, Chaudhary U. Study of virulence factors of uropathogenic Escherichia coli and its antibiotic susceptibility pattern. Indian $\mathrm{J}$ Pathol Microbiol 2014; 57:61-4. 
6. Hassan R, El-Naggar W, El-Sawy E, ElMahdy A. Characterization of some virulence factors associated with Enterobacteriaceae isolated from urinary tract infections in Mansoura Hospitals. N. Egypt J Med Microbiol 2011;20(2):9-17.

7. Pramodhini S, Umadevi S, Seetha KS. Detection of virulence determinants and its association with drug resistance in clinical isolates of Pseudomonas aeruginosa. Int $\mathbf{J}$ Res Med Sci 2016; 4:3917-23.

8. Seifu WD, Gebissa AD. Prevalence and antibiotic susceptibility of Uropathogens from cases of urinary tract infections (UTI) in Shashemene referral hospital, Ethiopia. BMC Infect Dis. 2018 Jan 10;18(1):30

9. Kline KA, Lewis AL. Gram-Positive Uropathogens, Polymicrobial Urinary Tract Infection, and the Emerging Microbiota of the Urinary Tract. Microbiol Spectr. 2016;4(2):10.

10. Todd, E. W. 1934. A comparative serological study of streptolysins derived from human and from animal infections, with notes on pneumococcal haemolysin, tetanolysin and staphylococcus toxin. J. Pathol. Bacteriol. 39:299-321.

11. Brock, T. D., B. Peacher, and D. Pierson. 1963. Survey of the bacteriocins of enterococci. J. Bacteriol. 86:702-707.

12. Su, Y. A., M. C. Sulavik, P. He, K. K Mikinen, P. Miikinen, S. Fiedler, R Wirth, and D. B. Clewell. 1991. Nucleotide sequence of the gelatinase gene (gelE) from Enterococcus faecalis subsp. liquefaciens. Infect. Immun. 59:415-420.

13. Das AK, Dudeja M, Kohli S, Ray P, Singh M, Kaur PS. Biofilm synthesis and other virulence factors in multidrug-resistant uropathogenic enterococci isolated in Northern India. Indian $\mathbf{J}$ Med Microbiol 2020;38:200-9.

14. Banerjee T, Anupurba S. Prevalence of Virulence Factors and Drug Resistance in Clinical Isolates of Enterococci: A Study from North India. J Pathog. 2015;2015: 692612

15. Ira P, Sujatha S, Chandra PS. Virulence factors in clinical and commensal isolates of Enterococcus species. Indian J Pathol Microbiol 2013;56:24-30

16. Thattil S J, Santhosh S. Prevalence of UTI in Different Age Groups in a Tertiary Care Hospital and their Antibiogram. International Journal of Contemporary Medical Research.2018;5(1);1-6

17. Shivani Raina and Dipender Kaur Najotra. Bacteriological Profile and Antibiogram of Uropathogens from a Tertiary Care Hospital: A Two-Year Retrospective Analysis. Int.J.Curr.Microbiol. App.Sci. 2019: 8(01): 1206-1212

18. Amit A. Rangari, Sachin Sharma, Nidhi Tyagi, Paramjit Singh, Garima Singh and Rameshwari Thakur. Antibiotic Susceptibility Pattern of Bacterial Uropathogens Isolated from Patients at a Tertiary Care Hospital in Western Uttar Pradesh of India. Int.J. Curr.Microbiol. App.Sci .2015: 4(10): 646-657

How to cite this article: Soujanya BR, Banashankari GS. Utility of chromogenic medium in characterization of enterococci in urinary tract infection and phenotypic detection of their virulence factors. Int $J$ Health Sci Res. 2021; 11(5): 278-283. DOI: https://doi.org/ 10.52403/ijhsr.20210544 